\title{
Sex Hormones Regulate Rat Hepatic Monocarboxylate Transporter Expression and Membrane Trafficking
}

Jieyun Cao, Michael Ng and Melanie Felmlee

Department of Pharmaceutics and Medicinal Chemistry, Thomas J Long School of Pharmacy and Health Sciences, University of the Pacific.

Received, October 23, 2017; Revised, November 8, 2017; Accepted, December 5, 2017; Published, December 13, 2017.

\begin{abstract}
Purpose: Monocarboxylate transporters (MCTs) are involved in the transport of monocarboxylates such as ketone bodies, lactate, and pharmaceutical agents. CD147 functions as an ancillary protein for MCT1 and MCT4 for plasma membrane trafficking. Sex differences in MCT1 and MCT4 have been observed in muscle and reproductive tissues; however, there is a paucity of information on MCT sex differences in tissues involved in drug disposition. The objective of the present study was to quantify hepatic MCT1, MCT4 and CD147 mRNA, total cellular and membrane protein expression in males, over the estrous cycle in females and in ovariectomized (OVX) females. Method: Liver samples were collected from females at the four estrous cycle stages (proestrus, estrus, metestrus, diestrus), OVX females and male Sprague-Dawley rats $(\mathrm{N}=3-5)$. Estrus cycle stage of females was determined by vaginal lavage. mRNA and protein (total and membrane) expression of MCT1, MCT4 and CD147 was evaluated by qPCR and western blot analysis. Results: MCT1 mRNA and membrane protein expression varied with estrous cycle stage, with OVX females having higher expression than males, indicating that female sex hormones may play a role in MCT1 regulation. MCT4 membrane expression varied with estrous cycle stage with expression significantly lower than males. MCT4 membrane expression in OVX females was also lower than males, suggesting that androgens play a role in membrane expression of MCT4. Males had higher membrane CD147 expression, whereas there was no difference in whole cell protein and mRNA levels suggesting that androgens are involved in regulating CD147 membrane localization. Conclusions: This study demonstrates hepatic expression and membrane localization of MCT1, MCT4 and CD147 are regulated by sex hormones. Sex differences in hepatic MCT expression may lead to altered drug disposition, so it is critical to elucidate the underlying mechanisms in the sex hormone-dependent regulation of MCT expression.
\end{abstract}

This article is open to POST-PUBLICATION REVIEW. Registered readers (see "For Readers") may comment by clicking on ABSTRACT on the issue's contents page.

\section{INTRODUCTION}

The liver is the major metabolic organ in the body with metabolism governed by drug metabolizing enzymes and transporter expression in hepatocytes. The solute carrier superfamily of transporters (SLC) are located on the basolateral membrane of hepatocytes and function to move drug substrates into and out of the cells with transport kinetics determined by substrate affinity and membrane transporter expression. These transporters may be a limiting factor in intracellular drug accumulation in hepatocytes, thereby determining the rate and extent of drug metabolism. Interindividual variations in transporter expression in the liver may contribute to differences in drug metabolism and systemic drug concentrations, leading to reduced efficacy or toxicity.

Monocarboxylate transporters (MCTs) are involved in the active transport of endogenous monocarboxylates such as ketone bodies, lactate and pyruvate, as well as drugs such as gammahydroxybutyrate, salicylic acid, nicotinate, nateglinide and valproic acid $(1,2)$. Proton-coupled monocarboxylate transporters are encoded by the SLC16A gene family. There are 14 members in the SLC16A gene family discovered by sequence homology (1). Currently, there are 5 members if the

Corresponding Author: Dr. Melanie A. Felmlee, Department of Pharmaceutics and Medicinal Chemistry, Thomas J. Long School of Pharmacy and Health Sciences, University of the Pacific, Stockton, CA, USA; Email: mfelmlee@pacific.edu 
family (MCT1-4, MCT7) demonstrated to be proton-dependent MCTs. Among those, MCT1 (SLC16A1), MCT2 (SLC16A7), and MCT4 (SLC16A3) are expressed in the liver of humans and rodents $(1,3)$. Recently, there is evidence that MCT7 (SLC16A6) facilitates the efflux of ketone bodies out of hepatocytes during fasting in a zebrafish model (4). Transport of substrates by MCTs can be bi-directional and is coupled with proton transport, and is driven by proton gradients instead of ATP (5). Ancillary proteins are involved in MCT membrane trafficking and functionality on the plasma membrane. CD147 (basigin) functions as an ancillary protein for MCT1, MCT3 (SLC16A8) and MCT4. Formation of MCT/CD147 heterodimers in the endoplasmic reticulum is important for efficient trafficking of the transporters to the plasma membrane $(6,7)$.

Previous studies have shown that MCT expression can be regulated by sex hormones. Enoki et al. (2006) reported that testosterone increased skeletal muscle MCT1 and MCT4 whole cell protein expression. However, MCT1 expression in the heart was not increased, suggesting MCTs are regulated in a tissue-specific manner (8). Additionally, rat Sertoli cells treated with 17betaoestradiol or dihydrotestosterone had decreased MCT4 mRNA levels (9). Hypophysectomized rats, with reduced endogenous testosterone levels, showed increased testicular MCT2 mRNA expression that was reversed with testosterone treatment ex vivo (10). Inhibition of estrogen related receptor alpha decreased MCT1 expression in MC4-L2 human breast cancer -bearing BALB/c mice indicating that estrogen may be involved in MCT1 regulation (11) These data suggest that MCTs are regulated by sex hormones in a tissuespecific manner; however, there is a lack of information in the literature evaluating the role of sex hormones on MCT expression in tissues involved in drug disposition.

The objective of this study was to quantitatively evaluate hepatic MCT1, MCT4 and CD147 mRNA, and total cellular and membrane protein expression in males, over the estrus cycle in female rats and in the absence of female sex hormones. We utilized five groups of female rats (proestrus, estrus, metestrus, and diestrus stages) and ovariectomized rats to evaluate MCTs and ancillary protein expression in rat liver over the estrus cycle as a response to the fluctuation in female sex hormones levels.

\section{METHODS}

Animals, Estrus Staging and Tissue Collection: Male and female Sprague Dawley (SD) rats and ovariectomized females were obtained from Envigo at 8 weeks of age, and were housed in a temperature controlled room with a 12-hour day/night lighting cycle. All rats were fed standard rat chow and water ad libitum. Estrus stages of female rats were monitored daily by vaginal lavage smear for $8-11$ days (at least two estrus cycle) between 10 am and $12 \mathrm{pm}$. Smears were stained with crystal violet (12) and imaged using a Keyence microscope. Vaginal lavage procedures were performed as previously described (13). Vaginal lavage was performed immediately before sacrificing to confirm their estrus stage, and each estrus stage evaluated represents an independent group of animals. Liver samples were collected ( $3-5$ per group; total of 24 animals) from all animals at 10 weeks of age following exsanguination under isoflurane anesthesia between $10 \mathrm{am}$ to $12 \mathrm{pm}$. Age-matched ovariectomized female samples were collected two weeks post-surgery. Liver samples were immediately snap frozen in liquid nitrogen and stored at $-80^{\circ} \mathrm{C}$ until analysis. All experiments were approved by the Institutional Animal Care and Use Committee at the University of the Pacific.

qPCR: Liver $(\sim 10 \mathrm{mg})$ was homogenized in lysis buffer and RNA was isolated using PureLink RNA Mini kit (Invitrogen) according to manufacturer's instructions. RNA concentration and purity was determined by Nanodrop (Thermofisher). RNA integrity was verified by RNA Flash Gels (Lonza) to visualize $18 \mathrm{~S}$ and $28 \mathrm{~S}$ rRNA. $2000 \mathrm{ng}$ of total RNA was added to each 40 $\mu 1$ cDNA synthesis reaction, which was reverse transcribed to cDNA using iScript Reverse Transcription Supermix (BioRad). Primer sequences, annealing temperatures and product sizes for quantitative real-time PCR are shown in Table $1(14,15)$. Primers were validated by running endpoint PCR with $250 \mathrm{nM}$ forward and reverse primers, $1 \mu 1$ of cDNA and iTaq Universal SYBR Green Supermix (BioRad) following the manufacturer's instructions. PCR product size was confirmed by running the amplicons on a $1 \%$ agarose gel (Figure 1). A TOPO TA Cloning kit (Invitrogen) was used to clone the amplicon into $\mathrm{E}$. coli and produce plasmid DNA containing the target sequence. The plasmid DNA was then digested using EcoR1 and run on a 1\% agarose gel 
to confirm successful insertion of the amplicon. Serial dilutions $\left(10^{-3}\right.$ to $\left.10^{-7}\right)$ were used as standards to confirm qPCR running conditions and efficiency. Quantitative analysis of MCT1, MCT4, and CD147 was performed with iTaq Universal SYBR Green Supermix as described above on CFX96 Connect (BioRad) with a standard curve run on every plate. The house-keeping gene 18S was evaluated for data normalization. The purity of all qPCR products was determined by melting curve. Data was quantified (in arbitrary units) based on the gene-specific standard curve.

Western blotting: Liver tissue was homogenized in $600 \mu \mathrm{l} \quad$ ice-cold radioimmunoprecipitation assay buffer (RIPA) containing Protease Inhibitor Cocktail (UltraCruz, Santa Cruz Biotechnology) and rotated for 15 minutes at $4^{\circ} \mathrm{C}$. The sample was centrifuged at $14000 \mathrm{x} \mathrm{g}$ for 15 minutes at $4^{\circ} \mathrm{C}$ to remove cell debris. The supernatant was retained for analysis of whole cell protein expression and stored at $-20{ }^{\circ} \mathrm{C}$ until analysis. Membrane bound protein was extracted from liver using a ProteoExtract Native Membrane Extraction kit (Millipore) following the manufacturer's instructions. The membrane and soluble fractions were retained, and stored at $-20^{\circ} \mathrm{C}$, and $\mathrm{Na}^{+} / \mathrm{K}^{+}$ATPase expression was evaluated by western blot to verify membrane isolation. Protein concentrations were determined using a Pierce BCA
Protein Assay Kit (ThermoFisher) with bovine serum albumin as the standard. Total whole cell protein $(15 \mu \mathrm{g})$ or membrane fraction $(5 \mu \mathrm{g})$ were mixed with $2 x$ Laemmli sample buffer (BioRad) and heated at $37^{\circ} \mathrm{C}$ for 30 minutes. Samples were separated at $200 \mathrm{~V}$ on $10 \%$ TGX Fast Cast gel (BioRad) and transferred to nitrocellulose membranes at $100 \mathrm{~V}$ for 25 minutes. Membranes were incubated in SEA Blocking buffer (ThermoFisher) for 1 hour at room temperature. Membranes were incubated with primary antibody overnight at $4^{\circ} \mathrm{C}$. Primary antibodies were rabbit anti-MCT1 (AB3540P, Millipore) at 1:1350 dilution, anti-MCT4 (AB3314P, Millipore) at 1:1000 dilution goat anti-CD147 (SC-9757, Santa Cruz) at $1: 1000$ dilution, rabbit anti- $\mathrm{Na}^{+} / \mathrm{K}^{+}$ ATPase (sc-28800, Santa Cruz) at 1:1000 dilution. Secondary antibodies were anti-goat or anti-rabbit IgG (Abcam) at 1:10,000 dilution, anti-rabbit (Santa Cruz) at 1:5000 dilution. The membranes were washed 3 times for $10 \mathrm{~min}$ in PBST and then incubated with secondary antibody for 1 hour at room temperature. The membranes were then washed $10 \mathrm{~min}$ for 3 times and incubated with Clarity ECL reagent (BioRad) for $2 \mathrm{~min}$. Protein expression was visualized using a Chemidoc Touch System (BioRad). Band density was determined using BioRad Image lab software with $\mathrm{Na}^{+} / \mathrm{K}^{+}$ ATPase as a loading control.

Table 1. Primer sequences, amplicon sizes, and annealing temperatures for qPCR assays $(14,15)$.

\begin{tabular}{ccccc}
\hline \multirow{2}{*}{ Gene } & F/R & Primer 5'-3' & $\begin{array}{c}\text { Size } \\
(\mathrm{bp})\end{array}$ & $\begin{array}{c}\text { Annealing } \\
\text { temperature }\end{array}$ \\
\hline \multirow{2}{*}{ MCT1 } & $\mathrm{F}$ & GCTGTCATGTATGCCGGAG & 204 & $60^{\circ} \mathrm{C}$ \\
& $\mathrm{R}$ & CAATCATAGTCAGAGCTGGG & & \\
CD147 & $\mathrm{F}$ & GGCACCATCGTAACCTCTGT & 211 & $60^{\circ} \mathrm{C}$ \\
& $\mathrm{R}$ & CAGGCTCAGGAAGGAAGATG & & \\
MCT4 & $\mathrm{F}$ & GCTGGCTATGCAGTATGGC & 185 & $60^{\circ} \mathrm{C}$ \\
& $\mathrm{R}$ & TTGAGAGCCAGACCCAAGC & & \\
\multirow{2}{*}{$18 \mathrm{~s}$} & $\mathrm{~F}$ & GTTGGTTTTCGGAACTGAGGC & 206 & $60^{\circ} \mathrm{C}$ \\
\hline
\end{tabular}

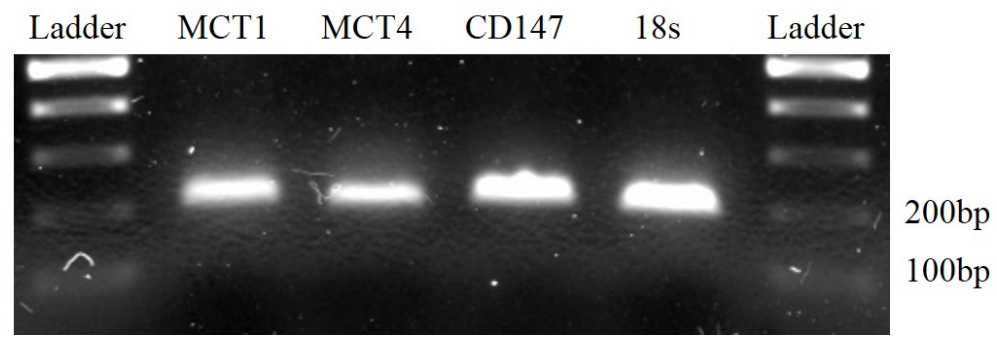

Figure 1. Primer validation for real time PCR. 
Data Analysis: Data are expressed as mean \pm standard deviation. Data was analyzed in GraphPad Prism 6 using a Kruskal-Wallis nonparametric oneway analysis of variance (ANOVA) with a Dunn's post hoc test. It was considered statistically significant when the $\mathrm{p}$ value was less than 0.05 . Correlation analysis in GraphPad Prism 6 was used to evaluate the relationships between mRNA expression and whole protein expression, and whole protein expression and membrane expression for all genes evaluated, and between CD147, MCT1, and MCT4 membrane expression.

\section{RESULTS}

Estrus cycle staging: After imaging, female estrus stages were identified by cell types in the smear (13). Vaginal lavage smears were evaluated daily to predict each rats' estrus stage on the day of tissue collection. The last vaginal lavage smear was acquired immediately prior to tissue harvesting and was used to confirm the estrus stage prediction. Representative imaging of the four stages are shown in Figure 2.

MCT1 expression: MCT1 mRNA expression varied significantly between groups $(\mathrm{P}=0.0199)$,

A

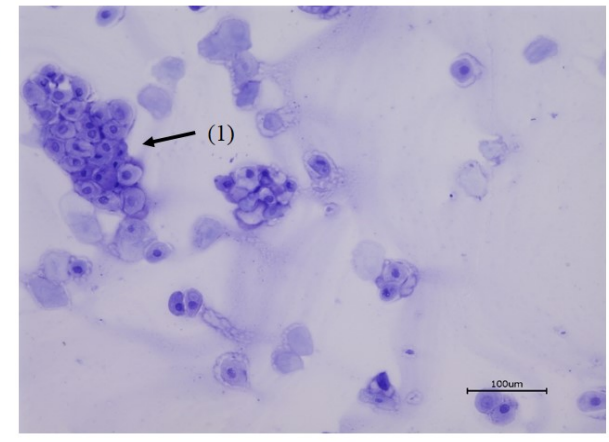

$\mathrm{C}$

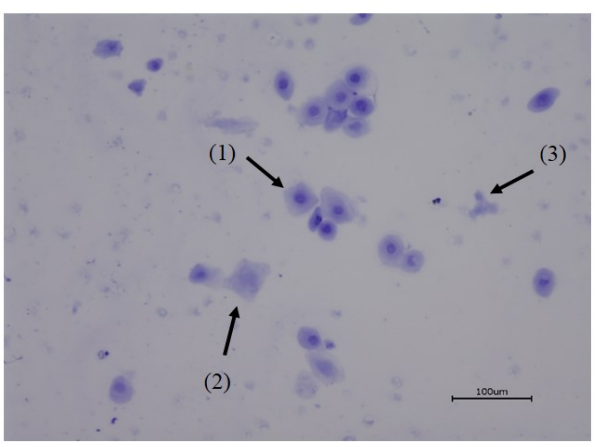

with lower mRNA expression in the presence of female sex hormones, as compared to males and ovariectomized females (Figure 3A). Female rats in the metestrus stage demonstrated statistically significant decreases in mRNA expression ( 90\%) compared to OVX rats. mRNA expression trended higher in OVX females as compared to males. Whole cell MCT1 expression varied based on sex $(\mathrm{P}=0.0632)$ and trended lower in all estrus stages, with ovariectomized females and males having the highest protein expression with greater variability than was observed for mRNA expression (Figure 3B). In contrast, membrane expression of MCT1 was highest in ovariectomized rats (approximately $50 \%$ higher than males) and differed based on female estrus cycle stages (Figure 3C), similar to the pattern observed in mRNA expression. MCT1 membrane expression varied significantly with sex $(\mathrm{P}=0.0086)$ with males and OVX females having higher MCT1 membrane expression than all female estrus cycle stages. In proestrus females, membrane MCT1 expression was significantly lower compared to ovariectomized females $(\sim 75 \%)$. Females in the estrus stage had higher expression than the other stages, but expression was still lower than OVX (55\%).

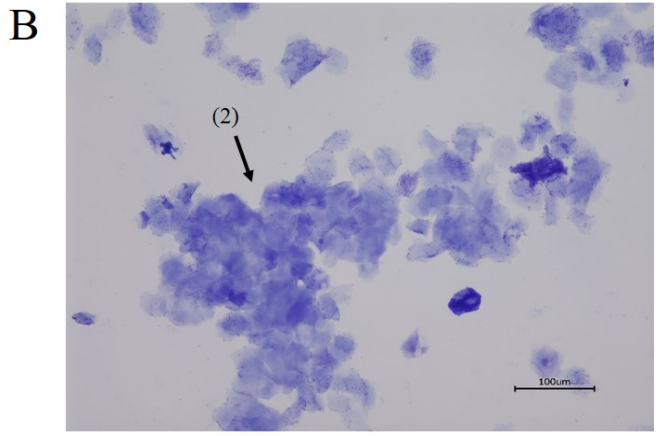

D

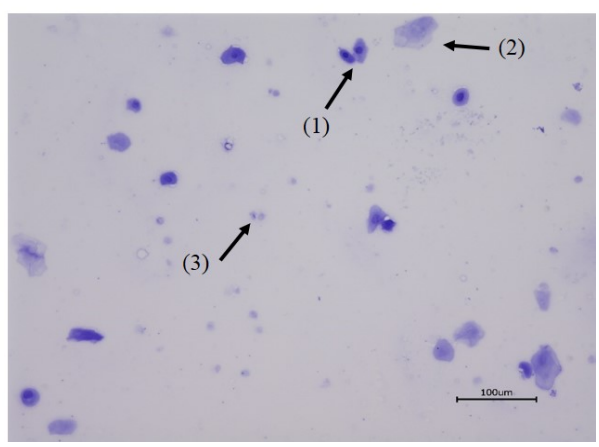

Figure 2. Representative vaginal smears for the four estrous cycle stages in female Sprague-Dawley rats at 20 times magnification. $A$. Proestrus characterized by large nucleated epithelial cells (1). $B$. Estrus characterized by large anucleated keratinized epithelial cells (2). C. Metestrus characterized by a combination of epithelial cells and neutrophils (3). $D$. Diestrus characterized by low cellularity, and a combination of neutrophils, small and large epithelial cells. 

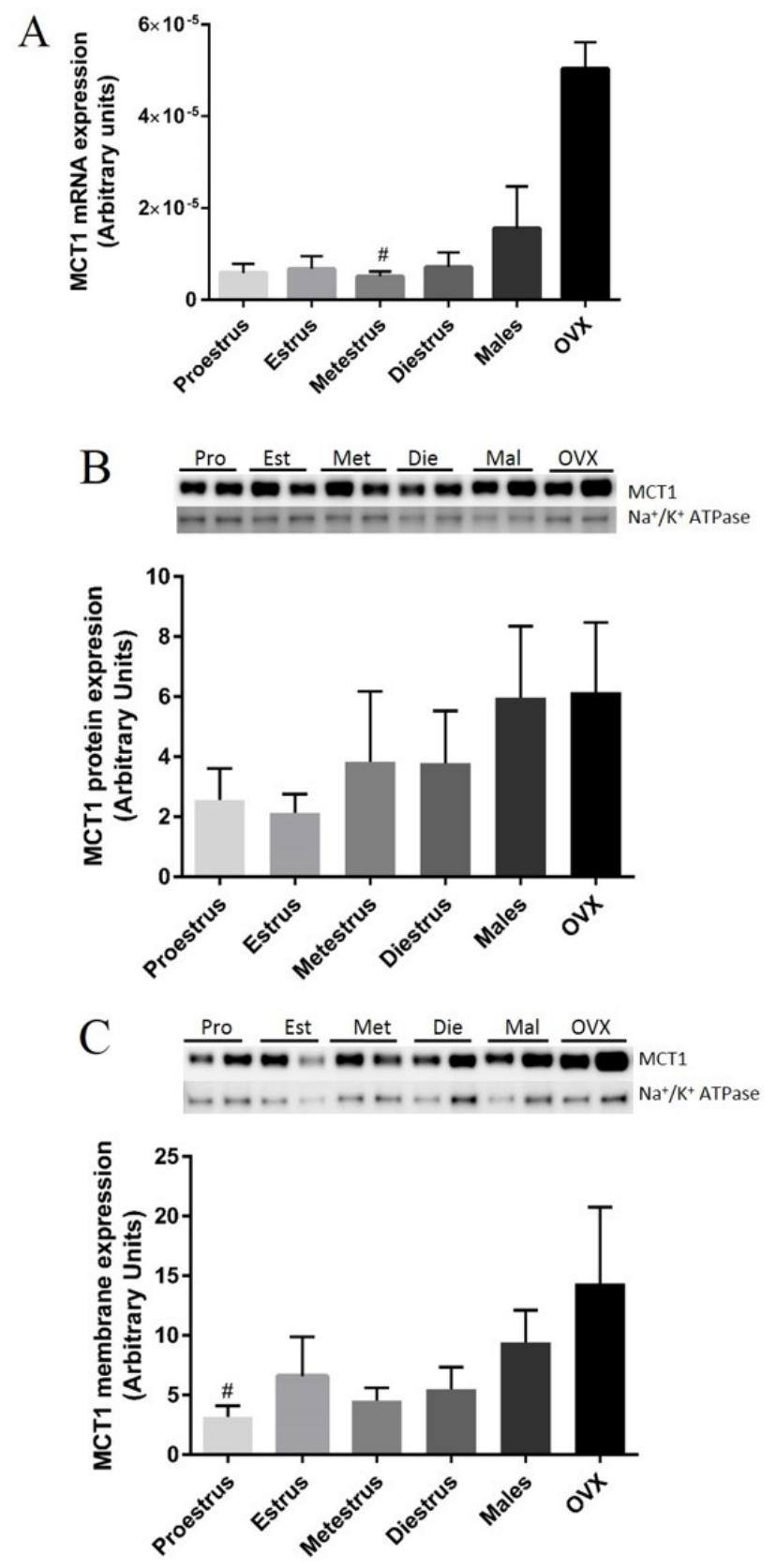

Figure 3. MCT1 mRNA, whole cell and membrane bound protein expression. $A$. Fold-change in MCT1 mRNA in Sprague Dawley rat liver. Data are presented as mean $\pm \mathrm{SD}$. $B$. Whole cell MCT1 protein expression in liver. Data normalized to $\mathrm{Na}^{+} / \mathrm{K}^{+}$ATPase expression, and presented as mean $\pm \mathrm{SD}$. C. MCT1 membrane protein expression. Data normalized to $\mathrm{Na}^{+} / \mathrm{K}^{+}$ATPase expression, and presented as mean $\pm \mathrm{SD} .{ }^{*} \mathrm{p}<0.05$ compared to male group. ${ }^{*} \mathrm{p}<0.05$ compared to OVX group. $(\mathrm{n}=3-5$ rats $)$.

MCT4 expression: MCT4 mRNA varied significantly between groups $(\mathrm{P}=0.0088$; Kruskal-
Wallis test) with all female estrous stages having lower expression as compared to males and OVX females (Figure 4A). MCT4 mRNA level was lower compared to MCT1 with $\mathrm{C}_{\mathrm{t}}$ values greater than 30 for MCT4. Whole cell MCT4 protein expression did not show significant difference between sexes or among female estrus cycle stages $(\mathrm{P}=0.6304)$, but this may have been due to the large degree of variability in expression. Membrane expression of MCT4 varied significantly with sex and estrus stage $(\mathrm{P}=0.0186 ;$ Kruskal-Wallis test). Membrane MCT4 expression was lower in females compared to males $(50-75 \%$ lower $)$ with statistically significant decreased expression observed in the proestrus stage. OVX females had lower membrane MCT4 when compared to males (approximately $60 \%$ lower).

CD147 expression: Expression of CD147, the ancillary protein for MCT1 and MCT4, is presented in Figure 5. There was no difference in mRNA expression of CD147 in the liver over the estrus cycle, or between males and ovariectomized females $(P=0.2443)$. Similarly, whole cell protein expression did not show any difference between sexes or ovariectomized females $(\mathrm{P}=0.8724)$. CD147 membrane expression was not statistically different between sexes $(\mathrm{P}=0.0749)$, but trended towards lower expression in all females. Membrane protein expression of CD147 was lower in all females when compared to males ( $60-65 \%$ lower), with expression in ovariectomized females similar to all estrus stages suggesting that androgens may be involved in membrane expression of CD147.

Correlation analysis: To examine the correlation between mRNA and whole protein expression, linear regression was performed on whole cell protein and mRNA expression data. MCT1, MCT4 and CD147 whole protein expression was not correlated with their mRNA expression indicating post-transcriptional regulation of expression (data not shown).

To evaluate if membrane expression correlated with whole protein expression, linear regression was used to analyze membrane and whole protein expression data. In Figure 6A, MCT1 membrane expression is positively correlated with MCT1 protein expression $\left(\mathrm{R}^{2}=0.36, \mathrm{p}<0.05\right)$, suggesting that membrane MCT1 expression is partially correlated with whole protein expression. MCT4 membrane expression was not correlated with MCT4 whole cell expression (Figure 6C), which 


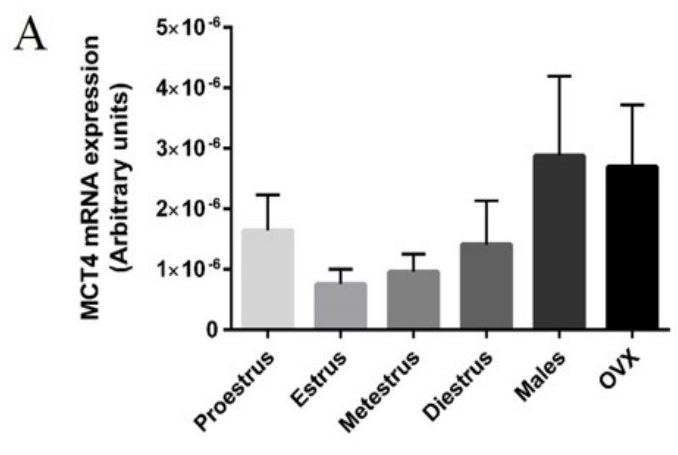

B Pro Est $\frac{\text { Met }}{\text { Die }} \frac{\text { Mal }}{\text { OVV }}$ $\mathrm{Na}+/ \mathrm{K}+\mathrm{ATPa}$

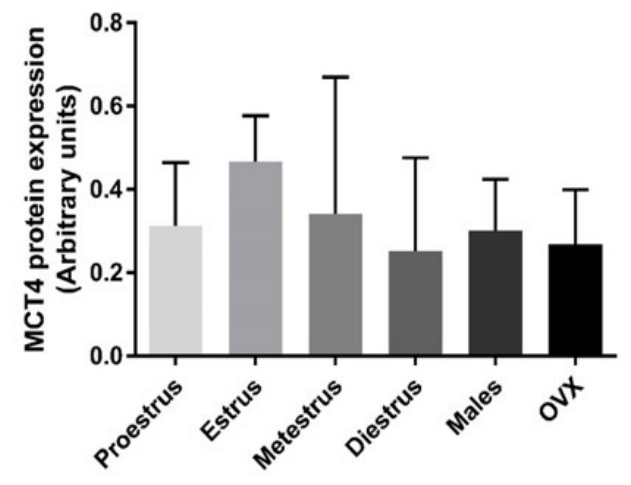

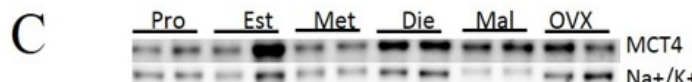

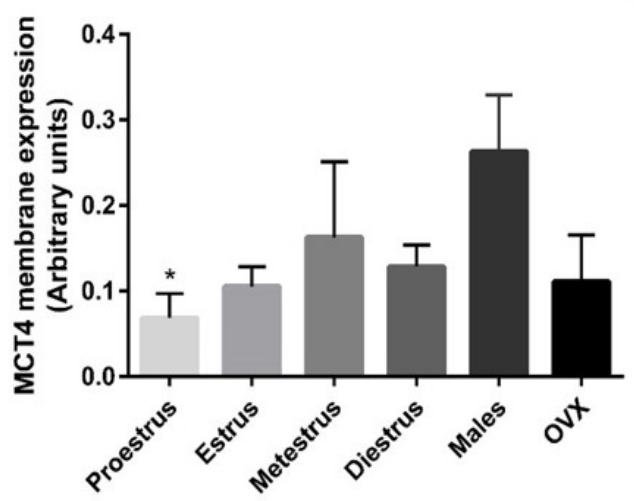

Figure 4. MCT4 mRNA, whole cell and membrane bound protein expression. A. Fold-change in MCT4 mRNA in Sprague Dawley rat liver. Data are presented as mean $\pm \mathrm{SD}$. $B$. Total MCT4 protein expression in liver. Data normalized to $\mathrm{Na}^{+} / \mathrm{K}^{+}$ATPase expression, and presented as mean $\pm \mathrm{SD}$. C. MCT4 membrane protein expression. Data normalized to $\mathrm{Na}^{+} / \mathrm{K}^{+}$ATPase expression, and presented as mean $\pm \mathrm{SD}$. ${ }^{*} \mathrm{p}<0.05$ compared to male group. ${ }^{*} \mathrm{p}<0.05$ compared to OVX group. $(\mathrm{n}=3-5$ rats $)$
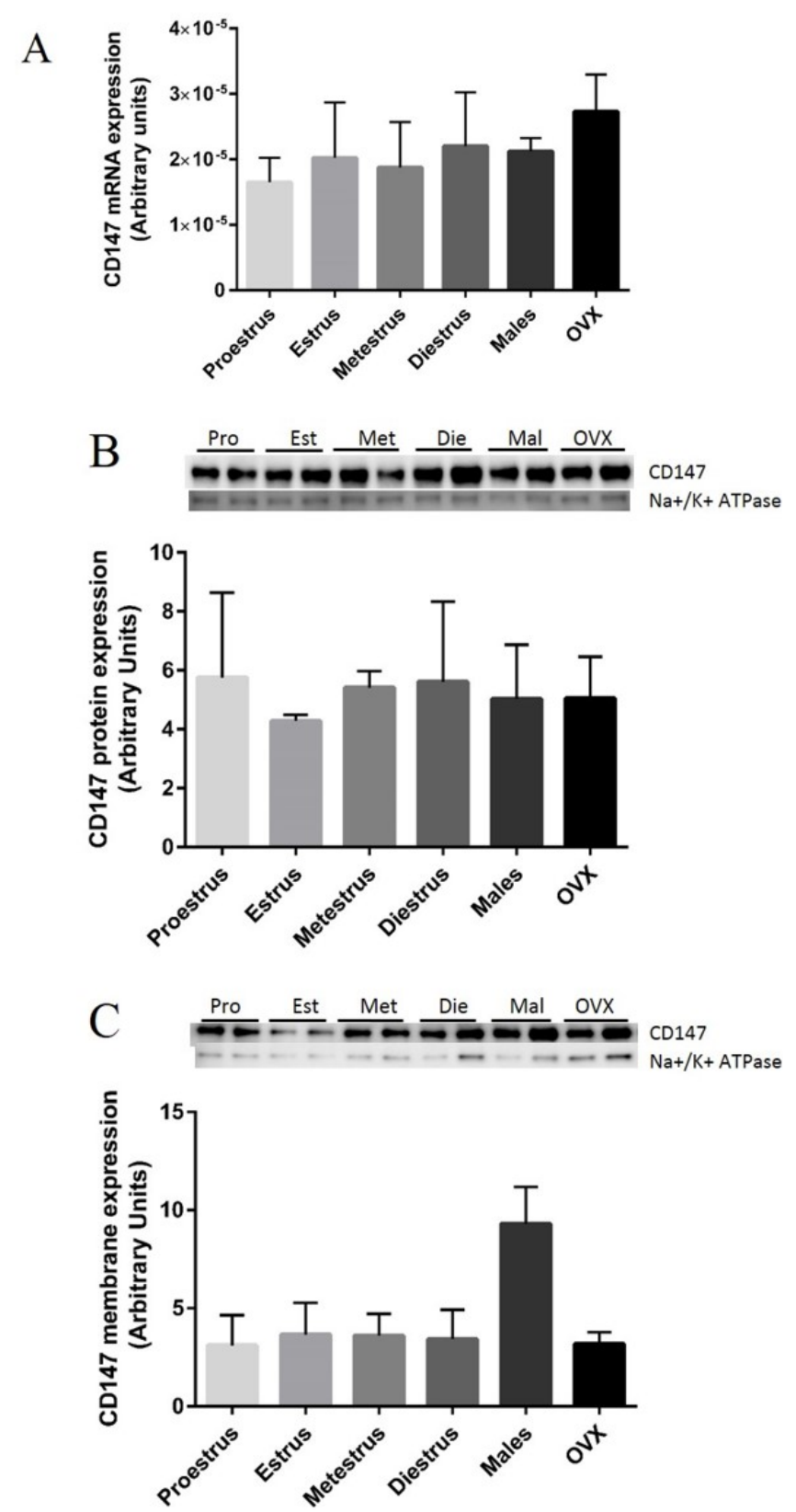

Figure 5. CD147 mRNA, whole cell and membrane bound protein expression. A. Fold-change in CD147 mRNA in Sprague Dawley rat liver. Data presented as mean $\pm \mathrm{SD}$. $B$. Whole cell $\mathrm{CD} 147$ protein expression in liver. Data normalized to $\mathrm{Na}^{+} / \mathrm{K}^{+}$ATPase expression, and presented as mean $\pm \mathrm{SD}$. C. CD147 membrane protein expression. Data normalized to $\mathrm{Na}+/ \mathrm{K}+$ ATPase expression, and presented as mean \pm SD. ${ }^{*} \mathrm{p}<0.05$ compared to male group. ( $\mathrm{n}=3-5$ rats). 
indicates that MCT4 membrane expression is dependent on post-translational regulation mechanisms. In addition, CD147 membrane expression does not correlate with CD147 whole protein expression (data not shown).

In order to further examine if $\mathrm{CD} 147$ is responsible for MCT1 and MCT4 membrane expression in the liver, correlations between membrane CD147 expression and membrane MCT1 and MCT4 expression were evaluated. MCT1 membrane protein expression showed a trend towards correlation with CD147 membrane protein (Figure 6B); however, the relationship was not statistically significant $\left(\mathrm{R}^{2}=0.09, \mathrm{p}>0.05\right)$ likely due to the presence of two outlying samples with very high MCT1 membrane expression. Both of these outliers were from OVX females, and with the OVX group removed there was a significant correlation between MCT1 and CD147 membrane expression $\left(\mathrm{R}^{2}=0.6789, \mathrm{p}<0.0001\right)$. This suggests that CD147 may not be solely responsible for MCT1 localization to the plasma membrane, and MCT1 membrane expression is exacerbated in the absence of female sex hormones. In Figure 6D, MCT4 membrane expression is significantly positively correlated with CD147 membrane
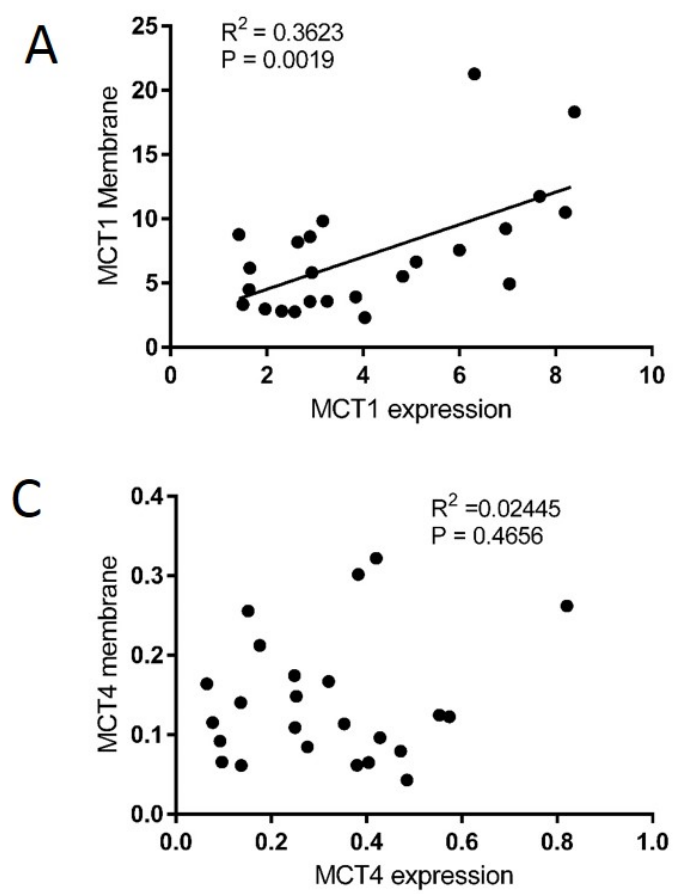

expression $\left(\mathrm{R}^{2}=0.40, \mathrm{p}<0.05\right)$. This indicates that CD147 is partially responsible for MCT4 membrane localization, and that other ancillary proteins may contribute to trafficking MCT4 to the plasma membrane.

\section{DISCUSSION}

Monocarboxylate transporters are involved in the distribution of nutrients and pharmaceutical agents in the liver. Transport capacity is governed by MCT membrane expression, and alterations in expression levels may lead to changes in systemic concentrations and hepatic metabolism. Previous studies have reported that MCTs are regulated by sex hormones in skeletal muscle and Sertoli cells $(8,9)$; however, there is a lack of information in tissues involved in drug disposition. The current study is the first analysis of sex hormone-dependent regulation of MCTs with respect to mRNA and protein expression, and membrane trafficking over the estrous cycle in female rats and in the absence of female sex hormones. We have demonstrated that female sex hormones are involved in the hepatic regulation of MCT1 mRNA and protein expression. In contrast, MCT4 and CD147
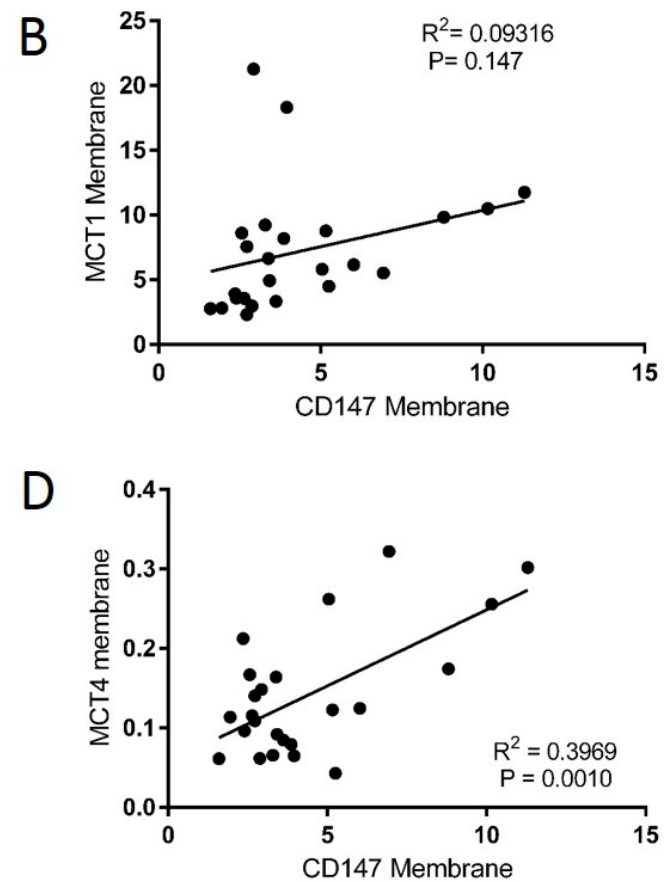

Figure 6. $A$. Correlation analysis between MCT1 membrane and whole cell protein expression. $B$. Correlation between CD147 membrane expression and MCT1 membrane expression. C. Correlation between MCT4 membrane protein and whole cell protein expression. D. Correlation between CD147 membrane expression and MCT4 membrane protein. $(\mathrm{n}=24$ rats) 
demonstrated consistent expression in the presence and absence of female sex hormones; however, males had higher membrane expression of MCT4 and CD147.

The estrous cycle in rats is typically 4 to 6 days long with female sex hormones fluctuating throughout the cycle. Diestrus can be between one and three days, with the other stages 24 hours or less in duration (13). In the present study, we evaluated the estrous cycle and collected liver samples during a defined time window to better capture all of the stages of the estrous cycle. Estradiol levels peak during the early portion of the proestrus stage, which often occurs in the morning, and then return to baseline within 24 hours (17). Luteinizing hormone, follicular stimulating hormone and progesterone levels peak later during proestrus and decrease to baseline at end of the proestrus day $(17,18)$. During estrus, estradiol levels increase to a lesser extent than during proestrus, and return to baseline during estrus (17). Baseline estradiol levels are observed in metestrus and diestrus stages (17). Progesterone levels are elevated again during the diestrus stage (18). Understanding the cycle-dependent changes in female sex hormonal levels will aid in identifying the individual hormones that may be involved in the regulation of MCTs and CD147.

Previous studies in rats have demonstrated sex hormone-dependent regulation of MCT1 mRNA and protein expression in a range of muscle types, focused on the role of testosterone. Testosterone treatment increased MCT1 mRNA and protein expression in muscle; however, the increases were muscle type dependent (8). Consistent with the observations in rats, sex differences in plasma lactate levels have been demonstrated following exercise in humans with women having lower plasma lactate levels than men suggesting there is increased MCT1 expression in men (19). We observed increased hepatic MCT1 mRNA and membrane expression in males and OVX females consistent with the testosterone-mediated increases demonstrated in rat muscle. Our results also suggest that female sex hormones may downregulate MCT1 mRNA expression and reduce membrane trafficking, supported by the estrous-cycle dependent fluctuations in MCT1 membrane expression. In contrast to the present results, Enoki et al. (2006) (8) observed decreased MCT1 protein expression in the heart with testosterone treatment suggesting that regulation of MCT1 by testosterone varies by tissue. Increased membrane MCT1 expression in males may result in increased hepatic uptake of MCT1 substrates, and future studies should evaluate hepatic transport of MCT1 substrates.

Testosterone-mediated increases in MCT4 mRNA and protein expression have been demonstrated in rat muscle with no correlation between mRNA and protein consistent with testosterone-mediated regulation of muscle MCT1 (8). In Sertoli cells, MCT4 mRNA expression did not differ from control when the cells were treated with estradiol or testosterone (20); however, Rato et al. (2012) (9) demonstrated decreases in MCT4 mRNA expression with testosterone treatment for 50 hours. We observed a significant difference in MCT4 mRNA expression between groups $(\mathrm{P}=$ 0.0088) with females of all estrous stages having lower expression than males and OVX females. However, we observed no difference from males with respect to total cellular protein expression in females over the estrous cycle or in the absence of female sex hormones. A significant difference was observed between groups for membrane MCT4 expression $(\mathrm{P}=0.0186)$ with all females having lower expression than males. Proestrus females had significantly lower membrane MCT4 expression as compared to males (post-hoc comparison). Membrane MCT4 expression did not differ between ovariectomized females and any of the estrous cycle stages suggesting that the membrane trafficking differences between males and females were mediated by testosterone. MCT4 is thought to function as an efflux transporter, therefore increased expression in males may result in decreased intracellular accumulation of MCT4 substrates in hepatocytes. Accumulation of compounds that are substrates of both MCT1 (uptake transporter) and MCT4 will be governed by the transporter with the highest abundance, which in the case of the liver is MCT1.

CD147, an ancillary protein for MCT1 and MCT4, is involved in their membrane trafficking, thus contributing to the amount of functional transporter in a given tissue. Previous studies have evaluated the role of estrogen receptors (ER) in CD147 regulation in reproductive tissues utilizing knock out mice, with ER $\alpha$ dependent regulation in the uterus and efferent ducts, and ER-independent regulation in other tissues (21). There is a paucity of information in the literature on sex hormonemediated regulation of CD147 in tissues involved in 
drug disposition. We have demonstrated differences in membrane expression of CD147, with males having higher expression than females, in the presence or absence of female sex hormones. No differences were observed in mRNA or total cellular protein expression of CD147 suggesting that testosterone plays a role in membrane trafficking and/or post-translational regulation. CD147 membrane expression correlated significantly with MCT4 membrane expression in all animals, while CD147 and MCT1 membrane expression showed a significant correlation when OVX females were removed from the analysis. These results suggest that both testosterone and female sex hormones may be involved in the regulation of membrane expression, and therefore functional MCT-mediated transport in the liver.

In summary, we have demonstrated sexhormone dependent regulation of MCT1 and MCT4 in the liver. MCT1 mRNA and protein expression demonstrated differences with estrous cycle stage and in the absence of female sex hormones, while MCT4 and CD147 membrane expression were independent of the estrous cycle and may be regulated by testosterone. Further studies are necessary to comprehensively evaluate the mechanisms underlying the sex hormone-dependent regulation of membrane expression of MCTs in hepatocytes, as well as the influence on substrate transport kinetics to predict the impact on hepatic drug disposition and pharmacokinetics.

\section{ACKNOWLEDGEMENTS}

Funding support was provided by the Nutting, Davidson and Sinai Foundation and University of the Pacific start-up funding for MAF.

\section{REFERENCES}

1. Morris ME, Felmlee MA. Overview of the protoncoupled MCT (SLC16A) family of transporters: characterization, function and role in the transport of the drug of abuse gamma-hydroxybutyric acid. AAPS J. 2008;10(2):311-21.

2. Kohyama N, Shiokawa H, Ohbayashi M, Kobayashi Y, Yamamoto T. Characterization of monocarboxylate transporter 6: expression in human intestine and transport of the antidiabetic drug nateglinide. Drug Metab Dispos. 2013;41(11):18837.

3. Schutkowski A, Wege N, Stangl GI, Konig B. Tissue-specific expression of monocarboxylate transporters during fasting in mice. PLoS One. 2014;9(11):e112118.

4. Hugo SE, Cruz-Garcia L, Karanth S, Anderson RM, Stainier DY, Schlegel A. A monocarboxylate transporter required for hepatocyte secretion of ketone bodies during fasting. Genes Dev. 2012;26(3):282-93.

5. Merezhinskaya N, Fishbein WN. Monocarboxylate transporters: past, present, and future. Histol Histopathol. 2009;24(2):243-64.

6. Kirk P, Wilson MC, Heddle C, Brown MH, Barclay AN, Halestrap AP. CD147 is tightly associated with lactate transporters MCT1 and MCT4 and facilitates their cell surface expression. EMBO J. 2000;19(15):3896-904.

7. Gallagher-Colombo S, Maminishkis A, Tate S, Grunwald GB, Philp NJ. Modulation of MCT3 expression during wound healing of the retinal pigment epithelium. Invest Ophthalmol Vis Sci. 2010;51(10):5343-50.

8. Enoki T, Yoshida Y, Lally J, Hatta H, Bonen A. Testosterone increases lactate transport, monocarboxylate transporter (MCT) 1 and MCT4 in rat skeletal muscle. J Physiol. 2006;577(Pt 1):43343.

9. Rato L, Alves MG, Socorro S, Carvalho RA, Cavaco JE, Oliveira PF. Metabolic modulation induced by oestradiol and DHT in immature rat Sertoli cells cultured in vitro. Biosci Rep. 2012;32(1):61-9.

10. Boussouar F, Mauduit C, Tabone E, Pellerin L, Magistretti PJ, Benahmed M. Developmental and hormonal regulation of the monocarboxylate transporter 2 (MCT2) expression in the mouse germ cells. Biol Reprod. 2003;69(3):1069-78.

11. Aveseh M, Nikooie R, Aminaie M. Exerciseinduced changes in tumour LDH-B and MCT1 expression are modulated by oestrogen-related receptor alpha in breast cancer-bearing BALB/c mice. J Physiol. 2015;593(12):2635-48.

12. McLean AC, Valenzuela N, Fai S, Bennett SA. Performing vaginal lavage, crystal violet staining, and vaginal cytological evaluation for mouse estrous cycle staging identification. J Vis Exp. 2012(67):e4389.

13. Cora MC, Kooistra L, Travlos G. Vaginal Cytology of the Laboratory Rat and Mouse: Review and Criteria for the Staging of the Estrous Cycle Using Stained Vaginal Smears. Toxicol Pathol. 2015;43(6):776-93.

14. Nikooie R, Rajabi H, Gharakhanlu R, Atabi F, Omidfar K, Aveseh M, et al. Exercise-induced changes of MCT1 in cardiac and skeletal muscles of diabetic rats induced by high-fat diet and STZ. J Physiol Biochem. 2013;69(4):865-77.

15. Liu Y, Liang WB, Zhang LS, Gao LB, Chen TY, Wang YY, et al. Expression of basigin in the early 
phase of acute myocardial ischemia in rats. Mol Med Rep. 2013;7(5):1494-500.

16. Livak KJ, Schmittgen TD. Analysis of relative gene expression data using real-time quantitative PCR and the 2(-Delta Delta C(T)) Method. Methods. 2001;25(4):402-8.

17. Barha CK, Galea LA. Influence of different estrogens on neuroplasticity and cognition in the hippocampus. Biochim Biophys Acta. 2010;1800(10):1056-67.

18. Emanuele MA, Wezeman F, Emanuele NV. Alcohol's effects on female reproductive function. Alcohol Res Health. 2002;26(4):274-81.

19. Cupeiro R, Gonzalez-Lamuno D, Amigo T, Peinado $\mathrm{AB}$, Ruiz JR, Ortega FB, et al. Influence of the
MCT1-T1470A polymorphism (rs1049434) on blood lactate accumulation during different circuit weight trainings in men and women. J Sci Med Sport. 2012;15(6):541-7.

20. Martins AD, Alves MG, Simoes VL, Dias TR, Rato L, Moreira PI, et al. Control of Sertoli cell metabolism by sex steroid hormones is mediated through modulation in glycolysis-related transporters and enzymes. Cell Tissue Res. 2013;354(3):861-8.

21. Chen L, Bi J, Nakai M, Bunick D, Couse JF, Korach $\mathrm{KS}$, et al. Expression of basigin in reproductive tissues of estrogen receptor-\{alpha\} or $-\{$ beta $\}$ null mice. Reproduction. 2010;139(6):1057-66. 